\title{
Trapping and Thrombectomy of Giant Thrombosed Vertebral Artery Aneurysm via Bilateral Subocciptal Approach - Technical
} Note

\author{
Pritam Gurung $^{1}$, Yoshihiro Kuga ${ }^{1}$, Yuji Kodama ${ }^{1}$, Katsushi Taomoto ${ }^{1}$, Hideyuki Ohnishi ${ }^{1}$ \\ ${ }^{1}$ Department of Neurosurgery, Ohnishi Neurological Center
}

Correspondence:

Dr. Pritam Gurung, MD., Ph.D.

Ohnishi Neurological center, Akashi, Hyogo, Japan

Email: preetamgurung@hotmail.com

\begin{abstract}
Background: Giant aneurysms arising from the vertebral artery (VA) are rare; they represent $4 \%$ to $6 \%$ of all intracranial giant aneurysm. The natural history of thrombosed aneurysms is extremely poor. Most such lesions progressively enlarge and result in irreversible progression of neurological deficits and fatal sequelae through resultant compression of the brainstem. We present the clinical experience of giant thrombosed vertebral artery aneurysm successfully treated via a bilateral suboccipital approach. A 62 -year-old woman presented with slight dysarthria and ataxia for one year. Neurological examination showed right lateral gaze nystagmus, bilateral absent corneal reflex, absent gag reflex, bilateral dysdiadochokinesia, poor right finger nose test, and slightly poor tandem gait. MRI showed a $27 \mathrm{~mm}$ giant thrombosed left VA aneurysm with brain stem compression. We performed trapping of the aneurysm and thrombectomy through a bilateral suboccipital approach. First, the distal portion was clipped from the left side. Next, the proximal portion was approached from the right side. Thrombectomy was performed and after shrinkage of the aneurysm, the clips were applied involving some part of the aneurysm just distal to PICA. Conclusion:The optimum treatment for aneurysm of this type is thought to be complete obliteration of the parent artery with trapping and thrombectomy to decompress the brainstem. Sometimes if PICA could not be preserved Occipital artery (OA)-PICA bypass should be considered.
\end{abstract}

Key words: Giant thrombosed aneurysm, Thrombectomy, Trapping, Vertebral artery

$\mathrm{G}$ iant aneurysms arising from the vertebral artery (VA) are rare; they represent $4 \%$ to $6 \%$ of all intracranial giant aneurysm. The natural history of thrombosed aneurysms is extremely poor. Most such lesions progressively enlarge and result in irreversible progression of neurological deficits and fatal sequelae through resultant compression of the brainstem. A variety of treatment modalities have been reported for this kind of aneurysm like proximal ligation, neck clipping, aneurysmectomy, trapping of the parent artery surgically, or endovascular coiling with/without bypass surgery, and flow diversion. ${ }^{1}$ We describe herein a case of giant thrombosed vertebral artery aneurysm successfully treated by trapping and thrombectomy via the bilateral suboccipital approach.

66

Date submitted: $28 / 05 / 2020$

Date accepted: 31/05/2020

\section{Case report}

A 62 -year-old woman presented to us with slight dysarthria and ataxia for one year. Neurological examination showed right lateral gaze nystagmus, bilateral absent corneal reflex, absent gag reflex, bilateral dysdiadochokinesia, poor right finger nose test, and slightly poor tandem gait. Magnetic resonance imaging (MRI) showed a $27 \mathrm{~mm}$ giant thrombosed left VA aneurysm with brain stem compression. In the T1 weighted image (Figure.1A), there is a slightly high signal to iso signal and T2-weighted MRI (Figure.1B and C) showed the mass lesion as mixed intensity. In the contrast MRI (Figure.1E), a partial contrast effect is recognized in the aneurysm wall. Plain CT head 
(Figure.1D) showed calcification of the aneurysm wall.

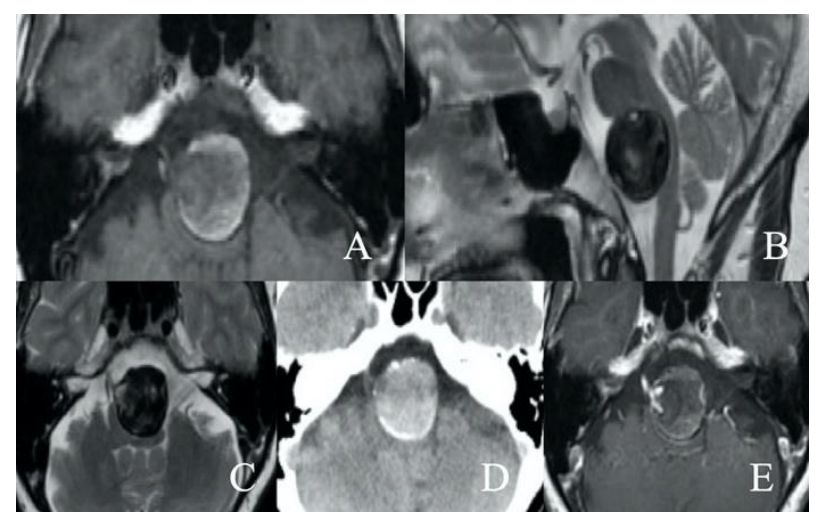

Figure.1. (A) Preoperative axial T1-weighted MRIshowing a 27 mm giant thrombosed left VA aneurysm with brain stem compression which was high signal to iso signal in intensity. (B) and (C) Sagittal and axialT2 weighted MRI showing the mixed intensity in the lesion. (D) Plain CT showing calcification of the aneurysm wall. (E) Axial T1 enhanced T1-weighted MRI showing partial contrast effect in the aneurysm wall.

Diagnostic cerebral angiography demonstrated the left vertebral artery occlusion distal to the PICA bifurcation (Figure.2A and B) and the right VA is compressed outward and backward (Figure.2C and D). The VA union was shifted to the right side by an aneurysm (Figure.2C).

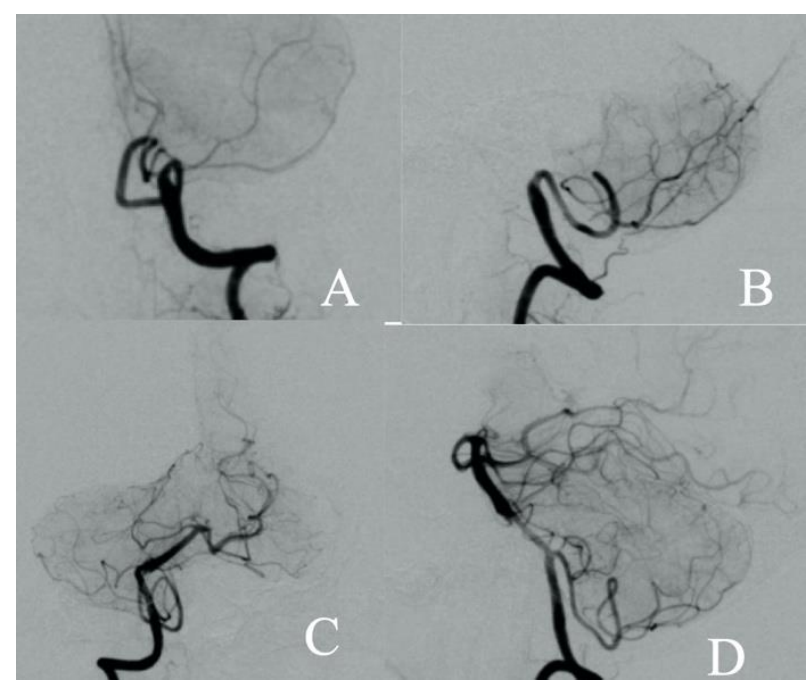

Figure.2. (A) and (B). Left vertebral artery imaging showing the vertebral artery occlusion distal to the PICA bifurcation. (C) and (D). Right VA imaging showing the vertebral artery is compressed outward and backward.

egneuro, Volume 02, Issue 02, 2020

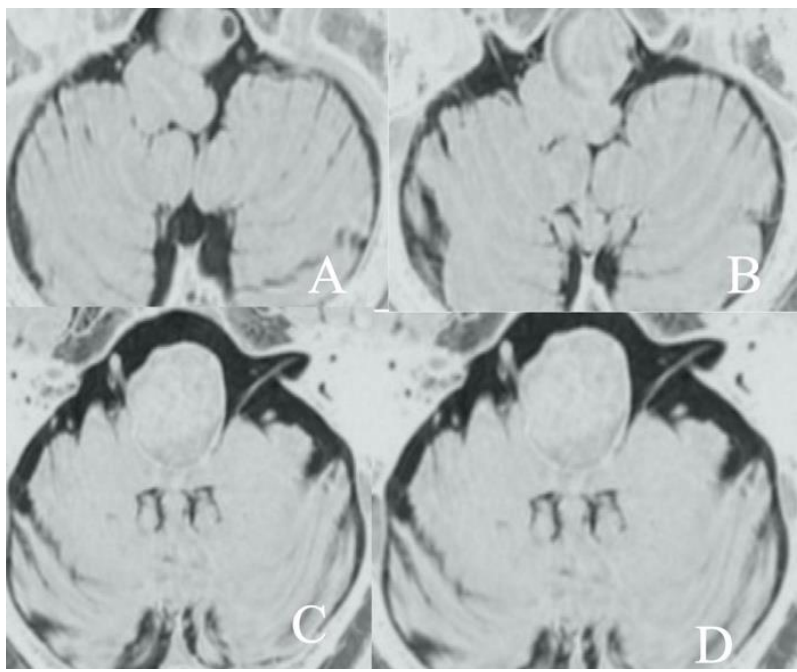

Figure.3. (A) Preoperative axial FIESTA MRI showing the proximal part of the aneurysm. (B) Bifurcation of PICA can be confirmed from the left side of the aneurysm wall. (C) When the aneurysm is traced to the distal end, the distal VA and VA union can be confirmed. (D)In the coronal image, it can be confirmed that PICA is branched from the proximal end of the left VA.

With the help of a fusion image (Figure.4A and B), we decided to perform trapping of the aneurysm and thrombectomy as a surgical strategy. The distal end of the aneurysm was on the right side of the midline and it was difficult to reach from the left side. Therefore, we decided on a bilateral suboccipital approach to trap the aneurysm proximally from the right side of the medulla and distally from the left side of the medulla (Figure.4C).

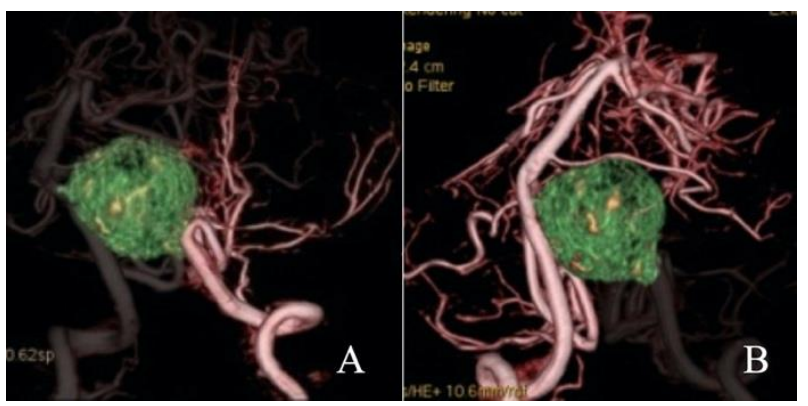

Figure .4. (A) This is a fusion image of 3D-DSA showing the PICA is branched from the vicinity of the proximal end of the left vertebral artery as seen in the MRI image described above. (B)The distal end of the VA is not visible but it is located on the right side of the midline.

The patient was placed in a prone position. A skin incision was made in the horse shoe shape extending to both sides (Figure.5A) and a large bilateral craniectomy was performed including $\mathrm{C} 1$ laminectomy. After a Y-shaped incision was made 
in the dura,the positional relationship between VA, PICA, aneurysm, and the lower cranial nerve was confirmed on both sides. We followed the left VA from the left side and applied a $10 \mathrm{~mm}$ straight clip to the distal end of the aneurysm (Figure.5C). Next, the proximal portion was approached from the right side where thrombosed giant aneurysm with the prominent left posterior inferior cerebellar artery (PICA) found to be running from the dome of the aneurysm (Figure.5B). From the right side, the aneurysm wall was cut with a blunt blade and the thrombus was removed as much as possible using an ultrasonic aspiration device. The aneurysm wall was coagulated to prevent blood flow from vasa vasorum and to reduce the mass.As estimated from the preoperative image, the calcification was strong and the thrombus was hard. After sufficient decompression was achieved, a $10 \mathrm{mmstraight}$ clip was applied to completely block the proximal end of the aneurysm preserving blood flow of PICA, but the blade did not reach and then, a $12 \mathrm{~mm}$ fenestrated clip was applied. An incision hole was also closed first with a $7 \mathrm{~mm}$ fenestrated clip and then applied a $7 \mathrm{~mm}$ straight clip. Fourclips were applied on the proximal side and one clip on the distal side (Figure. 5D). Finally, the patency of PICA confirmed by ICG video-angiography (Figure .5E). Thus, trapping of the aneurysm and thrombectomy was achieved.

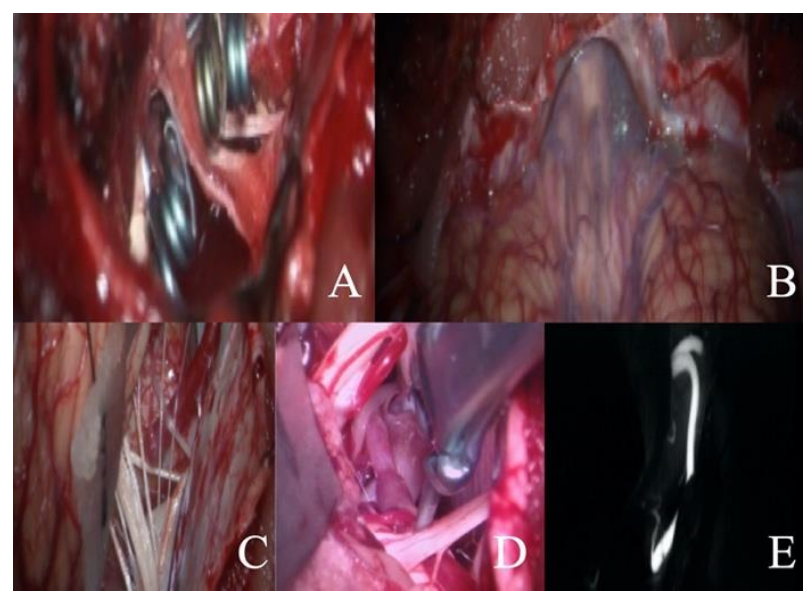

Figure .5. (A) Intraoperative finding of bilateral suboccipital craniectomy. (B) the proximal portion was approached from the right side where thrombosed giant aneurysm with the prominent left posterior inferior cerebellar artery (PICA) just running from the dome of the aneurysm was visualized. (C) The left VA on the distal side of the aneurysm was clipped from the left side, sparing perforating arteries and lower cranial nerves. (D) Four clips were applied on the proximal side and one clip on the distal side. (E) Patency of the PICA was confirmed with ICG video-angiography.
Postoperatively, consciousness was clear. Hoarseness and mild dysphagia remained, but the patient recovered without any effect on daily life. The patient was discharged 2.5 months after the operation. Postoperative MRI demonstrated brainstem without signs of compression (Figure.6B and C). On follow up images after 2.5 months of the first discharge, hydrocephalus was seen for which VP shunting was done.

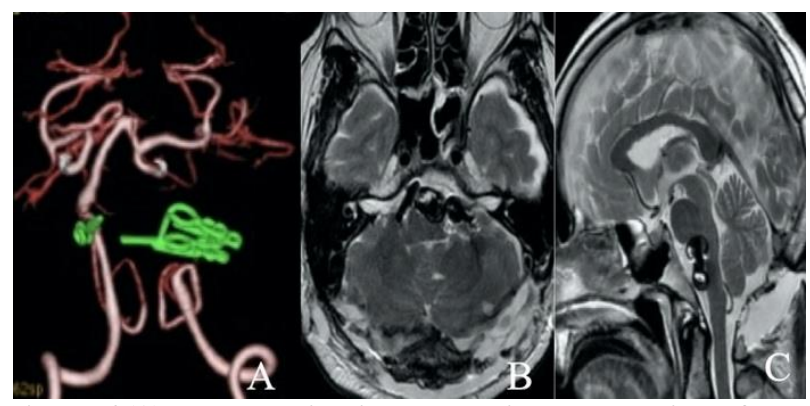

Figure 6.(A) Postoperative image showing the location of the clips.(B) \& (C) Postoperative MRI demonstrated brainstem without signs of compression.

\section{Discussion:}

There has been no consensus on the mechanism of the growth of the giant thrombosed vertebral artery aneurysm. The development of the intrathrombotic capillary channels may be an important factor in the growth of thrombosed giant aneurysm of the VA. ${ }^{2}$ The another growth mechanism is the role of vaso vasorum on the occluded parent artery in the dynamic process of neovascularization in the incomplete organization of thrombus around the packed coils. ${ }^{3}$ The continuous growth of giant aneurysm has been associated with hypertrophic vasa vasorum and subadventitial hemorrhages. ${ }^{4,5}$ Autopsy study suggests that simple dissection of the aneurysmal wall by residual blood flow in the lumen or by the blood flow in the parent artery through numerous clefts created abruptly in the organized thrombus near the wall of the distal neck may be involved in the growth and rupture of a thrombosed giant aneurysm of the VA. ${ }^{1}$

Neurological deficits due to the mass effect of a giant aneurysm are more severe in the posterior than in the anterior circulation. ${ }^{6,7}$ The basic goal of the surgery is to reduce the neurological deficits from their mass effect and preventing rupture. 
Various treatment modalities have been advocated for such aneurysm like proximal ligation, neck clipping, aneurysmectomy,trapping of the parent artery surgically or endovascular coiling with/without bypass surgery, coiling, and flow diversion. Bypass surgery is occasionally necessary when the flow from the contralateral vertebral artery is insufficient. When neurological deficits caused by the mass are minimal, even simple proximal ligation gives a satisfactory result. $8,9,10,11$ Aneurysmectomy is preferable to proximal ligation for partially thrombosed aneurysms in patients with severe neurological deficits. ${ }^{11}$ Clipping of the neck is possible only for saccular nonthrombosed aneurysms but giant aneurysms in this location are challenging because temporary clipping is often impossible in tight operating field and the aneurysms are usually atherosclerotic, fusiform or serpentine, with solid thrombosis. ${ }^{12}$ Clipping of giant thrombosed aneurysm is not feasible without trapping and thrombectomy. Ultralong clips of more than $30 \mathrm{~mm}$ blade length are useful. ${ }^{13}$ Straight and fenestrated clips are also essential for stability. ${ }^{14}$ In this case, we applied one straight clip in the distal side and four clips ( 2 straight, 2 fenestrated) in the proximal side.

Endovascular therapy has recently been advocated as a therapeutic alternative for giant aneurysms ${ }^{4,15,16}$ especially when the microsurgical approach is impossible due to deep anatomic location, tight posterior fossa, and short distance for trapping VA distal to the aneurysm. Coil embolization of the giant aneurysm without parent artery occlusion can be used for unclippable lesions, but this strategy can be associated with incomplete occlusion, rebleeding, regrowth, recanalization of aneurysms, coil compaction, and mass effect. ${ }^{7}$

A lateral suboccipital approach is commonly chosen for the surgical approach of VA aneurysms. Depend on the anatomical variations and tortuosity of the VA, transcondylar, transcondylar

fossa and extreme lateral inferior transtubercular exposures have been used as alternative approaches. $^{8}$ Midline suboccipital approach is basically used for medial cerebellar or brainstem lesion. ${ }^{17}$ Microscopic surgical field gets narrow when viewing the VA in front of the brainstem from the lateral side in midline suboccipital approach while the bilateral suboccipital approach will provide a sufficient lateral and caudal surgical field of view. ${ }^{5}$ In our case, we performed trapping of the aneurysm and thrombectomy through a bilateral suboccipital approach. First, the distal portion was clipped from the left side. Next, the proximal portion was approached from the right side where giant aneurysm can be visible with prominent left PICA just running from the dome of the aneurysm. In order to avoid brainstem injury by brain stem compression, aneurysmal debulking is considered safe. In this case, thrombectomy was performed and after shrinkage of the aneurysm, the clips were applied involving some part of the aneurysm just distal to PICA.

However, in cases where the calcification of the aneurysm is strong and hard, there is a possibility that the mass effect may not be alleviated in which partial resection of the aneurysm wall and removal of the thrombus may be necessary. In this case, partial calcification was observed, however, compression was reduced without damage to the brain stem.

\section{Conclusion:}

The optimum treatment for aneurysm of this type is thought to be complete obliteration of the parent artery with trapping and thrombectomy to decompress the brainstem. Sometimes if PICA could not be preserved Occipital artery (OA)-PICA bypass should be considered.

\section{References:}

1.YASUI T, SAKAMOTO H, KISHI H, KOMIYAMA M, IWAI Y, YAMANAKA K, NISHIKAWA M, NAKAJIMA H, KOBAYASHI Y, INOUE T. Rupture Mechanism of a Thrombosed Slow-growing Giant Aneurysm of the Vertebral Artery-Case Report-. Neurologia medico-chirurgica. 1998;38(12):860-4.

2. Nagahiro S, Takada A, Goto S, Kai Y, Ushio Y. Thrombosed growing giant aneurysms of the vertebral artery: growth mechanism and management. Journal of neurosurgery. 1995 May 1;82(5):796-801.

3. Iihara K, Murao K, Sakai N, Soeda A, Ishibashi-Ueda H, Yutani C, Yamada N, Nagata I. Continued growth of and increased symptoms from a thrombosed giant aneurysm of the vertebral artery after complete endovascular occlusion and 
trapping: the role of vasa vasorum: case report. Journal of neurosurgery. 2003 Feb 1;98(2):407-13.

4. Kiyohira M, Ishihara H, Oku T, Kawano A, Oka F, Suzuki M. Successful endovascular treatment for thrombosed giant aneurysm of the V1 segment of the vertebral artery: A case report. Interventional Neuroradiology. 2017 Dec;23(6):628-31.

5. Murai Y, Yamada T, Ishisaka E, Sato S, Matano F, Morita A. Bilateral suboccipital approach for a giant vertebral artery aneurysm. Journal of Clinical Neuroscience. 2017 Nov 1; $45: 315-8$.

6. Beck DW, Boarini DJ, Kassell NF. Surgical treatment of giant aneurysm of vertebral-basilar junction. Surgical neurology. 1979 Oct;12(4):283-5.

7. Drake CG. Giant intracranial aneurysms: experience with surgical treatment in 174 patients. Clin Neurosurg. 1979; 26:1295.

8. Day JD, Fukushima T, Giannotta SL. Cranial base approaches to posterior circulation aneurysms. Journal of neurosurgery. 1997 Oct 1;87(4):544-54.

9. Hosobuchi Y. Direct surgical treatment of giant intracranial aneurysms. Journal of neurosurgery. 1979 Dec 1;51(6):743-56.

10. Ishii R, Tanaka R, Koike T, Takeda N, Takeuchi S, Sasaki $\mathrm{O}$, Okada K. Computed tomographic demonstration of the effect of proximal parent artery ligation for giant intracranial aneurysms. Surgical neurology. 1983 Jun 1;19(6):532-40.

11. Sugita K, Kobayashi S, Takemae T, Tanaka Y, Okudera H, Ohsawa M. Giant aneurysms of the vertebral artery: Report of five cases. Journal of neurosurgery. 1988 Jun 1;68(6):960-6.

12. Wakui K, Kobayashi S, Takemae T, Kamijoh Y, Nagashima H, Muraoka S. Giant thrombosed vertebral artery aneurysm managed with extracranial-intracranial bypass surgery and aneurysmectomy: Case report. Journal of neurosurgery. 1992 Oct 1;77(4):624-7.

13. Sugita K, Kobayashi S, Inoue T, Takemae T. Characteristics and use of ultra-long aneurysm clips. Journal of neurosurgery. 1984 Jan 1;60(1):145-50.

14. Sugita K, Kobayashi S, Kyoshima K, Nakagawa F. Fenestrated clips for unusual aneurysms of the carotid artery. Journal of neurosurgery. 1982 Aug 1;57(2):240-6.

15. Capo G, Vescovi MC, Toniato G, Petralia B, Gavrilovic V, Skrap M. Giant vertebral aneurysm: a case report detailing successful treatment with combined stenting and surgery. Surgical neurology international. 2018;9.

16. Sluzewski M, Brilstra EH, Van Rooij WJ, Wijnalda D, Tulleken CA, Rinkel GJ. Bilateral vertebral artery balloon occlusion for giant vertebrobasilar aneurysms. Neuroradiology. 2001 Apr 1;43(4):336-41.
17. Bragg TM, Duckworth EA. Contralateral far-lateral approach for clipping of a ruptured vertebral artery-posterior inferior cerebellar artery aneurysm. Neurosurgical focus. 2008 Dec 1;25(6): E9. 